\title{
A Multi-agent Ant Colony Optimization Algorithm for Effective Vehicular Traffic Management
}

\author{
Saju Sankar $S^{1(\bowtie)}$ and Vinod Chandra S $S^{2}$ \\ 1 Department of Computer Engineering, Government Polytechnic College, Punalur, India \\ tkmce@rediffmail.com \\ 2 Computer Center, University of Kerala, Thiruvananthapuram, India \\ vinod@keralauniversity.ac.in
}

\begin{abstract}
An intelligent agent refers to an autonomous entity directing its activity towards achieving goals, acting upon an environment using data obtained with the help of a sensory mechanism. Intelligent agent software is a software system that performs tasks independently on behalf of a user in a networking environment based on user interface and past experiences. By the design of an intelligent sensing software program we can regulate the flow of traffic in a transportation infrastructure network. The problems leading to inefficiencies like loss of time, decrease in safety of vehicles and pedestrians, massive pollution, high wastage of fuel energy, degradation in the quality of life can be achieved by the optimized design. Ant Colony Optimization (ACO) has proven to be a very powerful optimization model for combinatorial optimization problems. The algorithm has the objective of regulating high real time traffic enabling every vehicle in the network with increased efficiency to minimize factors like time delay and traffic congestion.
\end{abstract}

Keywords: Ant colony optimization $\cdot$ Traffic control $\cdot$ Ant system $\cdot$ Pheromone

\section{Introduction}

Vehicle traffic control and monitoring systems are receiving much attention since several years because of large volume of traffic flows resulting in problems relating to ecology, economy and safety. Numerous vehicle control simulation techniques are adopted locally by means of bidirectional communication systems. This results in reduction of traffic blocks in various junctions by slowing down or accelerating the time delay factor. Genetic algorithms are adopted for the smooth passage of vehicular traffic as well as for emergency vehicles. The parameters of distance, speed and time are adjusted to the system needs. Currently no intelligence or optimization is adopted for the above scenario [1].

Intelligent agents are created using web technology techniques, maintaining a suitable database for past memory and by the use of appropriate programming languages thereby allowing the flow of traffic in modern developed and developing nations. Intelligent control methods have proven to be more advantageous than the currently used signal 
control methodology. To reduce the traffic congestion in cities, Intelligent Transportation Systems (ITS) has been adopted in developed countries.

Jinjian et al. studied the traffic control problem in an intersection traffic point by avoiding traffic lights [2]. The method assumes that all vehicles receive two-way communication from a cooperative control structure such that all vehicles are in communication zone. All safety constraints are applied in the above process. The Artificial Bee Colony (ABC) optimization algorithm is adopted with dynamic programming methods. Risikat et al. proposed an adaptive dynamic scheduling algorithm based on $\mathrm{ABC}$ for controlled vehicle traffic [3]. The model minimized the average waiting time at the intersection and leads to reduced vehicle queues. The Artificial Bee Colony (ABC) algorithm is a population based meta-heuristic optimization which was inspired by the fore aging behavior of the honey bee swarm [4]. The ABC algorithm is employed to determine the appropriate green light duration for each of the roads forming a four-way intersection.

D Alves et al. introduced a static traffic prediction model which is based on Ant Dispersion Routing algorithm (ADR) and it is designed to reduce the travelling time and for improving the efficiency of the traffic system [5]. The process does not consider dynamic factors such as network pruning and flow optimization. Rutgar et al. [6] proposed an Ant Colony Optimization [ACO] algorithm for extracting solutions to problems related to distributed structures. The congestion is avoided by way of intelligent systems and future estimations.

Soner et al. explained an ACO based algorithm (ACROSES and ACOTRANS) for a group of signalized junctions by means of a software tool TRANSYT-F [7]. It uses Performance Index as the metric and the results shows better compared to genetic and hill climbing algorithms. Jayachithra et al. [8] proposed an ACO algorithm based on Vehicle Ad Hoc Network (VANET) for finding the less and highly congested paths and thereby reducing the total travelling time in the network. It uses ACO protocols AODV, DSDV and DSR which results in parameters like throughput and end to end delay. David et al. applied the ACO technique to reduce the user waiting time at traffic intersections by implementing a rank based ant system and the control signals are monitored by heuristic information [9].

In this paper, we propose an ant colony optimization based traffic management, with a quality software intensive system which works on the principle of depositing pheromones en route. The intensity of pheromone deposit and updating features are the main principle of Ant Colony Optimization (ACO) used in traffic management. ACO outperforms all the other optimization techniques like $\mathrm{ABC}$ used in traffic control and management by way of intelligent ant systems.

\section{ACO Based Traffic Management System}

ACO is a probabilistic technique which searches for optimal path. The behavior of ants is seeking a shortest path between their colony and source of food. It is a Meta heuristic optimization. The path is discovered by pheromone deposits when the ants move at random. More pheromone deposit on a path increases the probability of the path being followed. The path is selected based on the maximum pheromone deposit from start node and the path is analyzed for optimality. 
ACO shows great performance in network routing and traffic scheduling. In the ant colony system algorithm, the original ant system was modified in three aspects i) the selection of path is biased towards the path with a large volume of pheromone ii) while building a solution, ants change the pheromone level of the edges they are selecting by applying a local pheromone updating rule iii) at the end of each iteration, only the best ant is allowed to update the pheromone value by applying a modified global pheromone updating rule.

\subsection{ACO Algorithm for Traffic Management}

The ACO algorithm is an agent based algorithm which gives optimized path in a large network [10]. In this study we have derived Procedure for dynamic pheromone update and detection quality of agent based computational technique that gives an optimal solution. The procedure that uses ant colony agent based computation is,

Input:

Set of ants $A=a_{1}, a_{2} \ldots, a_{n}$.

Set of paths $P=p_{1}, p_{2}, \ldots p_{m}$.

$A_{n}=$ vehicle incoming to the queue in each path.

$\mathrm{q}=$ queue length, $\mathrm{d}=$ decay factor, $\mathrm{p}=$ pheromone intensity value.

$\mathrm{t}=$ time delay, initially set to $1 \mathrm{~min}$.

Output:

Time period ' $\mathrm{t}$ ' when the queue is maximum.

Method:

1. Initialize pheromone trails in each path, initially set to zero.

2. Arrival of ants in each path ( $\mathrm{p}_{1}$ to $\mathrm{p}_{\mathrm{m}}$ ).

3. Case 1: Cross junction

a. All left paths are always open except pedestrian crossings.

b. For paths 1 to $\mathrm{m}$, generate ant movements marked by pheromone deposit.

c. Update pheromone value in all paths.

$$
\tau_{i j}=(1-\rho) \tau_{i j}+\Delta \tau_{i j}
$$

d. Generation of traffic movement based on pheromone value.

e. On movement, pheromone value evaporation.

f. Fixing priority path based on probability.

$$
P_{i j}^{k}(t)=\left\{\begin{array}{cc}
\frac{\left[\tau_{i j}(t)\right]^{\alpha} *\left[\eta_{i j}\right]^{\beta}}{\sum_{l \in \mathcal{N}_{i}^{k}}\left[\tau_{i l}(t)\right]^{\alpha} *\left[\eta_{i l}\right]^{\beta}}, & j \in \mathcal{N}_{i}^{k} \\
0, & j \notin \mathcal{N}_{i}^{k}
\end{array}\right.
$$

g. Repeat until threshold value of pheromone intensity is reached.

h. Go to step 3, until all paths are optimized in a round robin manner. 
4. Case 2: repeat step 3 for "Y" junction and "T" junction.

5. Calculation of waiting time for all three intersection methods.

6. End.

\subsection{ACO Based Traffic Control Model}

ACO is an algorithm based on the behavior of the real ants in finding the shortest path from a source to the food. Ant colony is used to determine the optimal path from source to destination. Routing is the act of moving information across a network from a source to destination. Ants deposit a certain amount of pheromone on its path. It is similar to common memory [11].

The artificial ants simulated as vehicles are driven by a probability rule to sequentially choose the solution components that make use of pheromone deposit intensities and heuristic information. Once an ant has constructed a solution, the ant evaluates the full solution to be used by the algorithm's next step in determining how much pheromone to deposit. The probability rule guides ant movement through a local decision policy that essentially depends on pheromone deposit, decay/evaporation information and heuristic information [12].

ACO can be applied to optimize traffic network problems. In this problem the vehicles are simulated as artificial ants from the source along the traffic network. The routes are estimated by the amount of pheromone deposits along the designated path. The entire path is monitored by the usage of image sensors and pheromone sensors [13]. While entering a cross junction, whether it is 4 way or 3 way, all the left free paths are open irrespective of the pheromone deposit.

In the case of all other path ways, the permission is based on the maximum queue length of the traffic. The queue length is estimated by the pheromone sensors for estimating which path has the highest pheromone deposit value. Ants simulated as vehicles are assigned weights depending upon the type - heavy, medium, light. They are assumed pheromone intensity weights as 2,1 and 0.5 . All the pheromone updates or scheduled information are stored in a database server for future predictions. The possible permissible paths of the vehicles are simulated in a graphical way whether they are in a 4-way junction or 3 way junctions ( $\mathrm{Y}$ or $\mathrm{T}$ ).

As soon as the most prioritized lane of vehicles passes the cross junction, the pheromone values for that vehicles are updated as zero or the decay factor for that lane is zero. The next path with highest pheromone deposit will be the next one who gets signaling. The process will continue in a round robin fashion until all the paths will be exhausted one after the other.

\section{Results and Discussion}

We have obtained data for testing the ACO algorithm from various government organizations like National Transportation Planning and Research Centre (NATPAC) and Center for Development for Advanced Computing (CDAC). The received data contained collection of Traffic Census on National Highways in the states of Kerala, Tamil Nadu, 
and Karnataka which involves around 300 major junctions. The simulation parameters are type of vehicle, count of incoming vehicles, volume of pheromone deposit, decay/evaporation factor, priority, waiting time in each queue and the average waiting time. The analysis of data gives a measure of the average waiting time of each vehicle obtained by the ACO optimization.

The following assumptions were made in our proposed method.

1. There are three separate queues in each direction.

2. Vehicles will be stationed as per choice of direction in each queue.

3. Measurement of pheromone deposit is read by way of intelligent sensors.

4. The data obtained from sensors are collected in a server for immediate processing and follow up of permission for traffic control.

5. Pedestrian crossing is also considered.

ACO optimization imparted in the simulation.

1. If the pheromone deposit intensity is equal, then there is no need of optimizing.

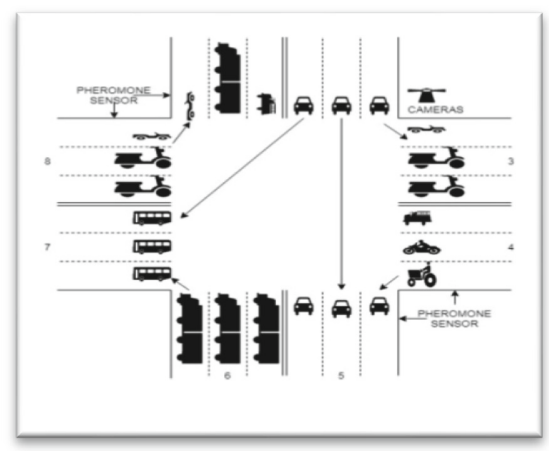

(a)

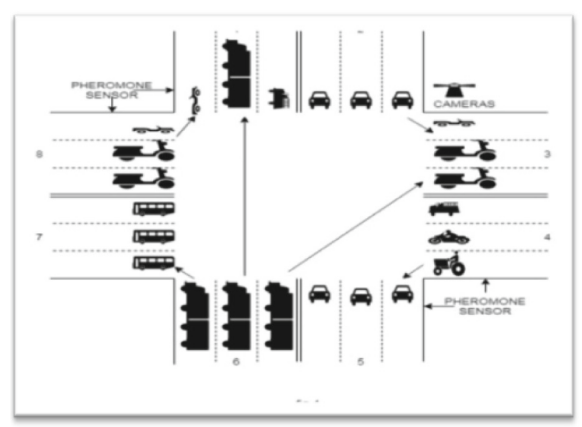

(c)

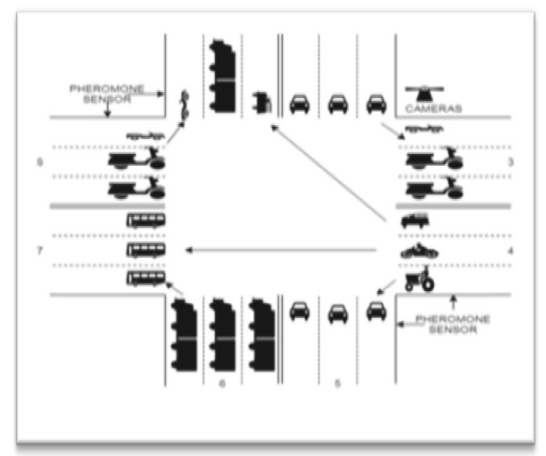

(b)

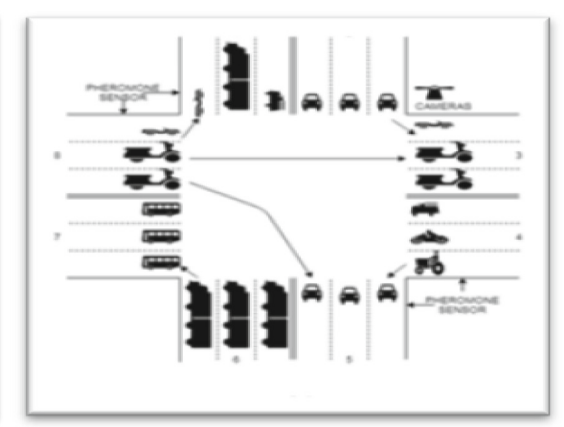

(d)

Fig. 1. (a) shows the permissible path ways from lane 2 to other routes. (b) shows the permissible pathways from lane 4 to all other routes. (c) the permissible path ways from lane 6 to other routes. (d) shows the permissible pathways from lane 8 to all other routes. 
2. Usually all paths are given a time duration of one minute.

3. If the pheromone intensity is varied considerably or unequal in one or more paths, then optimization is done by having the less pheromone path's passage time is deducted and assigned to the needy paths thereby optimizing time.

4. When there are pedestrians, then all the four paths are optimized.

5. When a priority vehicle arrives, then the path's pheromone value is reset and the current priority vehicle is set the maximum value and reset the path to the old value.

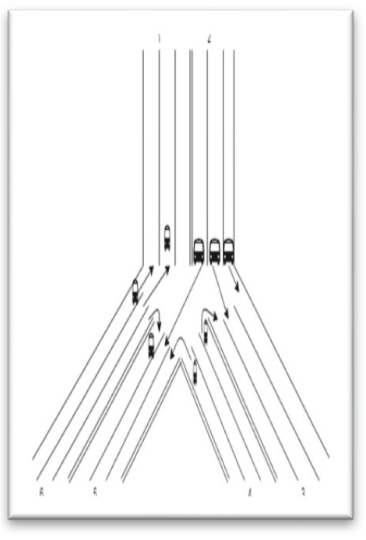

(a)

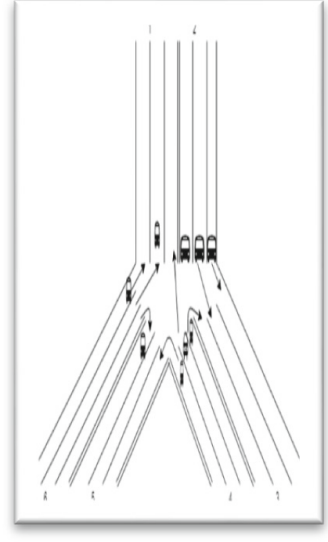

(b)

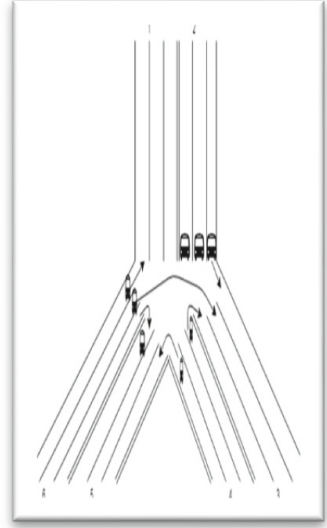

(c)

Fig. 2. (a) shows the permissible path ways from lane 2 to other routes, (b) shows the permissible pathways from lane 4 to all other routes. (c) shows the permissible pathways from lane 6 to all other routes.

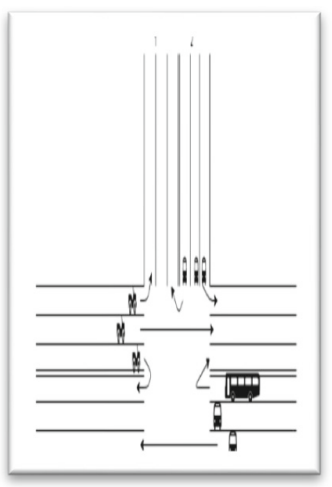

(a)

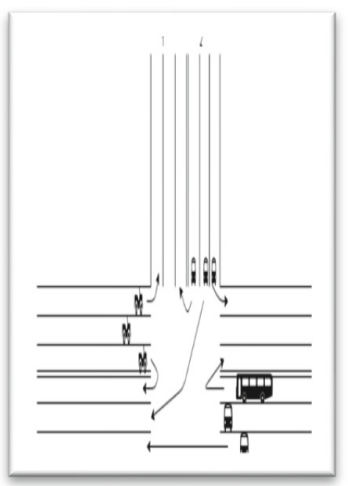

(b)

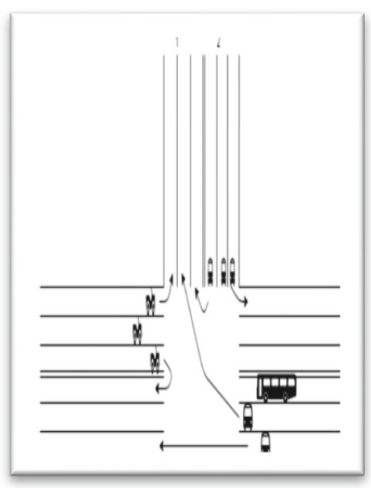

(c)

Fig. 3. (a) shows the permissible path ways from lane 2 to other routes, (b) shows the permissible pathways from lane 4 to all other routes and (c) shows the permissible pathways from lane 6 to all other routes. 


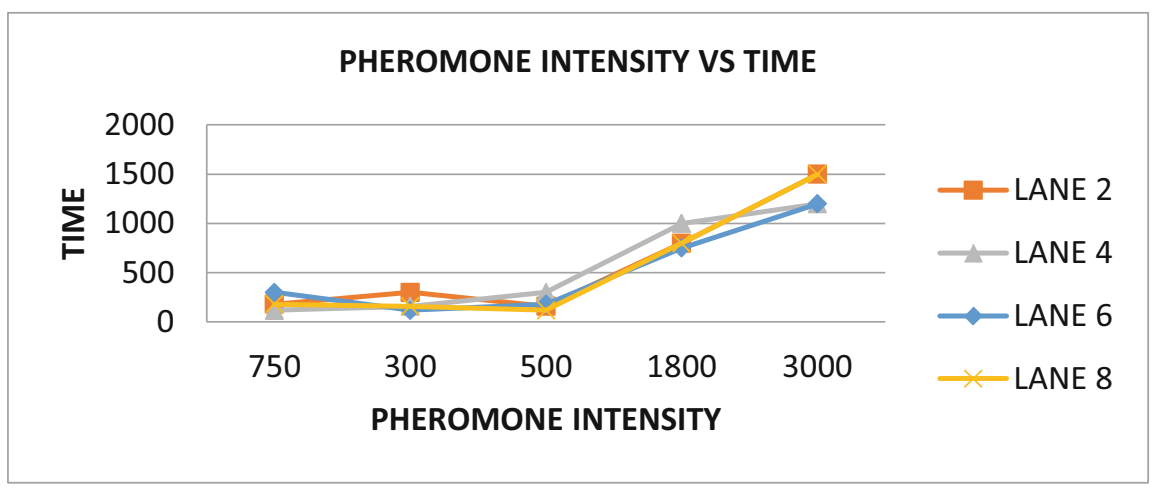

Fig. 4. Graph showing the time delay for each of the four lanes.

Figure 1(a) through Fig. 1(d) illustrate the adopted method of measuring the pheromone intensity in a cross junction for each of the lanes 2, 4, 6, 8 and their priority fixing mechanism. The lanes $1,3,5,7$ are for outgoing traffic only.

In the case of ' $Y$ ' junction, Fig. 2(a) through Fig. 2(c) illustrates the adopted method of measuring the pheromone intensity for each of the lanes 2, 4, 6 and the priority fixing mechanism. The lanes 1, 3, 5 are for outgoing traffic only.

In the case of ' $T$ ' junction, Fig. 3(a) through Fig. 3(c) illustrates the adopted method of measuring the pheromone intensity for each of the lanes 2, 4, 6 and the priority fixing mechanism. The lanes $1,3,5$ are for outgoing traffic only

Traffic management being a real time application, the simulation was done on an intelligent software system implementing the features of the ant system. All the vehicle movements are represented by a graph connecting all the lanes in different directions. There will be a threshold sealing of pheromone deposit in a single lane to avoid priority assignment only for a single lane so that all lined vehicles will be permitted to pass through in a random fashion.

A graphical analysis was conducted for the ACO optimization. The graph has pheromone deposit intensity in the $\mathrm{X}$-axis and time delay in seconds as the $\mathrm{Y}$-axis. It clearly shows how the time stamp is mapped with pheromone deposit intensity for unequal traffic and how the time slice is effectively utilized (Fig. 4).

The results clearly emphasize that ACO based optimization reduced the waiting time delay to a minimum in all the lanes of all directions. While comparing to the traffic optimization done by ABC optimization model and routing algorithms, the ACO model produced better traffic management for varying flow of vehicles and priority is also implemented for emergency vehicles.

\section{Conclusion}

In our model, we concluded through our research and simulation, ACO techniques are highly suitable for effectively controlling huge traffic in cities due to its dynamic nature of traffic management. This is efficient because ant works as intelligent agents and the pheromone deposit is mapped with movements. The algorithm also prioritizes 
emergency vehicles in a traffic junction. The decay or evaporation of pheromone is also taken into consideration for scheduling traffic. Usage of intelligent sensors and by the dynamic nature of the software made the management of traffic outperforming all the other optimized techniques. The model can be applied to a chain of interconnected junctions thereby minimizing the traffic congestion in a large network.

Acknowledgement. Authors would like to thank National Transportation Planning and Research Centre (NATPAC), who provided us real time data of most of the cities in India needed for the simulation, experimental activities and their routing problems.

\section{References}

1. Kelly, M., Di Marzo Serugendo, G.: A decentralised car traffic control system simulation using local message propagation optimised with a genetic algorithm. In: Brueckner, S.A., Hassas, S., Jelasity, M., Yamins, D. (eds.) ESOA 2006. LNCS (LNAI), vol. 4335, pp. 192-210. Springer, Heidelberg (2007). https://doi.org/10.1007/978-3-540-69868-5_13

2. Li, J., et al.: Cooperative traffic control based on the artificial bee colony. J. Eng. Res. Appl. 6(12(Part-I)), 46-55 (2016)

3. Adebiyi, R.F.O., et al.: Management of vehicular traffic system using artificial bee colony algorithm. Int. J. Image Graph. Sig. Process. 9(11), 18-28 (2017)

4. Saritha, R., Vinod Chandra, S.S.: Multi modal foraging by honey bees toward optimizing profits at multiple colonies. IEEE Intell. Syst. 34(1), 14-22 (2018)

5. Alves, D., et al.: Ant colony optimization for traffic dispersion routing. In: Proceedings of the 13th International IEEE Conference on Intelligent Transportation systems (ITSC), Portugal (2010)

6. Claes, R., Holvoet, T.: Cooperative ant colony optimization in traffic route calculations. In: Demazeau, Y., Müller, J., Rodríguez, J., Pérez, J. (eds.) Advances on Practical Applications of Agents and Multi-Agent Systems. AINSC, vol. 155, pp. 23-34. Springer, Heidelberg (2012). https://doi.org/10.1007/978-3-642-28786-2_3

7. Haldenbilen, S., et al.: An Ant Colony Optimization Algorithm for Area Traffic Control. INTECH (2013)

8. Jayachithra, N., et al.: Shortest path using ant colony optimization in VANET. Int. J. Eng. Res. Technol. (IJERT), RTICCT (2017)

9. Renfrew, D., et al.: Traffic Signal Optimization using Ant Colony algorithm. In: The International Joint Conference on Neural Networks (IJCNN), Australia (2012)

10. Vinod Chandra, S.S.: Smell detection agent based optimization algorithm. J. Inst. Eng. (India) Ser. B 97, 431-436 (2016). https://doi.org/10.1007/s40031-014-0182-0

11. Vijayalaxmi, et al.: Ant colony optimization technique in network routing problem - a simulation study. In: International Conference on Innovations in engineering and Technology, ICIET 2013, Bangkok, Thailand, 25-26 December 2013 (2013)

12. Karova, M., et al.: Ant colony optimization algorithm for traffic flow estimation. In: International Conference on Computer Systems and Technologies - CompSysTech 2017, Ruse, Bulgaria (2017)

13. Kumar, D., et al.: Improvement in traffic light system with ant colony optimization technique. Int. J. Eng. Res. Appl. 3(6), 1744-1749 (2013). ISSN 2248-9622 